\title{
ON THE CASTELNUOVO-SEVERI INEQUALITY FOR RIEMANN SURFACES
}

\author{
Robert D. M. Accola
}

\begin{abstract}
Some consequences of equality in the Castelnuovo-Severi inequality are discussed. In particular, it is shown that if a Riemann surface of genus ten, $W_{10}$, admits four coverings of tori, each in three sheets, then $W_{10}$ admits an elementary abelian group of order 27. By previous work this last result is then characterized by the vanishing of certain thetanulls. An elementary discussion of the direct product of monodromy groups is an essential part of the proofs.
\end{abstract}

\section{Introduction}

The inequality of Castelnuovo-Severi is as follows $[2,4]$. Let $W_{g}$, a compact Riemann surface of genus $g$, cover two Riemann surfaces, $W_{h}$, genus $h$, in $m$ sheets, and $W_{k}$, genus $k$, in $n$ sheets, so that the two coverings admit no common non-trivial factorization. (If $m$ and $n$ are primes this will always be the case.) Then the Castelnuovo-Severi inequality (CSI) states:

$$
g \leq m h+n k+(m-1)(n-1)
$$

A natural (and venerable) question is whether there is a Riemann surface $W_{\ell}$, covered by $W_{h}$ in $n$ sheets, and covered by $W_{k}$ in $m$ sheets, so that the resulting square diagram commutes. In the case of equality in the CSI a theorem of Castelnuovo gives much more information, so that in this case the question should be easier to answer. For $h=k=0$ the answer is: sometimes yes and sometimes no (Section 8). So perhaps the question should be that of finding necessary and sufficient conditions for the existence of such a $W_{\ell}$, which must necessarily be the Riemann sphere $\mathbf{P}^{1}$. We shall give a characterization when $h=k=1, m=3$ and $n$ an odd prime (Theorems 4.5 and 4.6). A more general situation is covered in Theorem 4.2 where $h$ and $k$ are large compared to $m$ and $n$.

This investigation had its origin in a $W_{10}$ covering several tori each in three sheets, where this situation is characterized by the existence on $W_{10}$ of certain half-canonical linear series [3]. The goal was to show that if $W_{10}$ covered four

AMS Mathematical Subject Classification. Primary 14H30, Secondary 30F10.

Received June 13, 2005. 
such tori then $W_{10}$ admitted an automorphism group isomorphic to an elementary abelian group of order 27 (Section 7). It turns out that a substantial part of the proof can be carried out when 3 is replaced by any odd prime $p$, and perhaps this clarifies the proof. Section 5 carries out that part of the proof with 3 replaced by $p$. Section 6 gives examples.

By Riemann's solution to the Jacobi inversion problem the existence of halfcanonical linear series on $W_{10}$ is equivalent to the vanishing of the theta function for the Jacobian of $W_{10}$ at certain half-periods (vanishing thetanulls) [5]. Thus the existence on a $W_{10}$ of such a group of order 27 is characterized by the vanishing of certain thetanulls.

This paper deals with a subject and methods available to mathematicians in the early part of the last century, if not earlier. Considering the extensive literature already existing at that time, the author really has no idea whether some if not all of the material in this paper is already part of the literature. If a reader knows of any reference in the literature that pertains to the work here presented, the author would be very grateful to know of it.

\section{Preliminary results}

First some notation. $\quad W_{g}$ will always stand for a compact Riemann surface with the lower case subscript denoting its genus, in this case $g . W_{F}$ will always denote a compact Riemann surface with a finite set of points, $P t_{F}$, deleted with the upper case subscript denoting its fundamental group (in this case $F$ ) after a suitable base point has been chosen. $W_{F}^{*}$ will denote the compact Riemann surface obtained by adding the points $P t_{F}$ to $W_{F}$; that is $W_{F}^{*}-P t_{F}=W_{F} . W_{F}$ and $W_{F}^{*}$ have the same genus. Occasionally we will use both kinds of notation, $W_{g}$ and $W_{F}^{*}$, for the same compact Riemann surface.

If $A$ is a subgroup of $F$ of finite index $m$, then after suitable base points have been chosen the topological mapping $\pi_{A F}: W_{A} \rightarrow W_{F}$ is defined. (We will always assume, without further comment, such suitable base points chosen so that coverings of Riemann surfaces with subgroup subscripts $F, A, B, A \cap B, \ldots$, will correspond to the inclusion relations of the subgroups indicated; e.g., $W_{A \cap B} \rightarrow$ $W_{A}$.) The covering map can be extended to $\pi_{A F}: W_{A}^{*} \rightarrow W_{F}^{*}$ where $P t_{A}$ maps onto $P t_{F}$. (If $W_{A}^{*}$ has genus $h$ and $W_{F}^{*}$ has genus $\ell$ we may denote $\pi_{A F}$ by $\pi_{h \ell}$.

Let $A x_{1}, A x_{2}, \ldots, A x_{m}$ be a coset decomposition of $F$. These cosets correspond to the points on $W_{A}$ above the base point for $F$. The monodromy map $\mu: F \rightarrow S_{m}$ (the symmetric group on the first $m$ digits) is as follows: $\mu(f)(i)=j$ if $A x_{i} f=A x_{j}$. (Here we will make no notational distinction between a path $f$ and its homotopy equivalence class.) Let $A_{0}$ denote the kernel of $\mu$. Then $F / A_{0}$ is isomorphic to $\operatorname{Im}(\mu)$, the monodromy group of the covering $\pi_{A F}$ denoted $\operatorname{Mono}\left(W_{A} / W_{F}\right)$. By definition it is the monodromy group of the ramified covering $\pi_{A F}: W_{A}^{*} \rightarrow W_{F}^{*}$, and may be denoted $\operatorname{Mono}\left(W_{A}^{*} / W_{F}^{*}\right)$ or $\operatorname{Mono}\left(W_{h} /\right.$ $\left.W_{l}\right)$. If $f(\in F)$ "circles" a point $t \in P t_{F}$ let $\alpha_{1}, \alpha_{2}, \ldots, \Sigma \alpha_{i}=m$, be the multiplicities of the points in $P t_{A}$ above $t$. By renumbering the $x$ 's we may assume that $x_{1} f^{\alpha_{1}} x_{1}^{-1}, x_{2} f^{\alpha_{2}} x_{2}^{-1}, \ldots$ are paths in $A$ which circle the points in $P t_{A}$ above 
$t$. If $f \in A_{0}$ there are $m$ such paths and no ramification occurs above $t$ in $\mathrm{Pt}_{A}$.

Now assume $A$ and $B$ are two different subgroups of $F$ of indices $m$ and $n$ respectively. Also assume that $F=A B$ and that $A \cap B$ has index $m n$ in $F$. If $m$ and $n$ are prime this will be the case. Let $B y_{1}, B y_{2}, \ldots$ be another coset decomposition of $F$.

Now suppose that $f(\in F)$ circles $t \in P t_{F}$. Suppose that $x f^{\alpha} x^{-1}$ (now dropping subscripts) circles a point $a$ in $P t_{A}$ above $t$, and suppose that $y f^{\beta} y^{-1}$ circles a point $b$ in $P t_{B}$ above $t$, where $\alpha$ and $\beta$ are minimum positive integers with these properties. Since $(A x \cap B y) f^{\gamma}=A x f^{\gamma} \cap B y f^{\gamma}$ we see that if $\gamma=$ 1.c.m. $[\alpha, \beta]$ then $(A x \cap B y) f^{\gamma}=(A x \cap B y)$ where $\gamma$ is the minimum positive integer with this property. There is at least one point $c$ in $P t_{A \cap B}$ where $c$ is above $a, b$, and $t$, and the ramification at $c$ for the covering $\pi_{A \cap B, A}$ is $[\alpha, \beta] / \alpha$. The number of such $c$ 's is g.c.d. $(\alpha, \beta)$.

Lemma 2.1. Assume the above discussion. If $f \in A_{0}$ then the multiplicities of the points above any such $a$ in the covering $\pi_{A \cap B, A}$ are the same as those above $t$ in the covering $\pi_{B, F}$. The covering $\pi_{A \cap B, B}$ is unramified over any point in $P t_{B}$ above $t$.

Lemma 2.2. Assume the above discussion. Suppose above $a \in P t_{A}$ the covering $\pi_{A \cap B, A}$ is unramified and that $x f^{\alpha} x^{-1}(\in A)$ circles a. Then $f^{\alpha} \in B_{0}$.

Proof (Exponentiation by an element in $F$ denotes conjugation.).

$$
x f^{\alpha} x^{-1} \in \bigcap\left\{(A \cap B)^{g} \mid g \in A\right\}=A \cap \bigcap\left\{B^{g} \mid g \in A\right\}
$$

But $F=A B$ so $\bigcap\left\{B^{g} \mid g \in A\right\}=\bigcap\left\{B^{g} \mid g \in F\right\}=B_{0}$. Since $B_{0}$ is normal in $F$ the result follows.

In this paper we have assumed (and will continue to assume) many wellknown facts from elementary group theory. However, for future reference we wish to draw attention to the following.

Lemma 2.3. Let $G$ be a finite group, the direct product of two normal subgroups, $M$ and $N$. Let $A$ be a normal subgroup of $G$ so that $A \cap M=$ $A \cap N=\langle e\rangle$. Then $A$ is central in $G$, and $A$ is isomorphic to a subgroup of $M$ which is central in $M$.

Lemma 2.4. With the same hypotheses as in Lemma 2.3, assume further that $A$ and $M$ are isomorphic to subgroups of $S_{p}$ ( $p$ an odd prime) whose orders are divisible by $p$. Then $A$ and $M$ are isomorphic to $Z_{p}$ (Cyclic group of order $p$ ).

(since the centralizer of $Z_{p}$ in $S_{p}$ is $Z_{p}$ itself.) 
Lemma 2.5 [2, p 23-25]. Let $\pi_{g h}: W_{g} \rightarrow W_{h}$ be an m-sheeted covering, and let $\pi_{g k}: W_{g} \rightarrow W_{k}$ be an $n$-sheeted covering where the two coverings admit no common non-trivial factorization. Then the number of pairs of points, counted with multiplicity, common to fibers of the two maps is finite. (This is the statement that the algebraic curve $W_{g}$ lying on the surface $W_{h} \times W_{k}$ has a finite number of singularities.)

Lemma 2.6 (Castelnuovo [2, p 26]). Suppose for $W_{g}, W_{h}$, and $W_{k}$ in Lemma 2.5 we have equality in the Castelnuovo-Severi inequality. Then for $x_{1}$ and $x_{2}$ in $W_{h} \pi_{g k}\left(\pi_{g h}^{-1}\left(x_{1}\right)\right)$ is linearly equivalent to $\pi_{g k}\left(\pi_{g h}^{-1}\left(x_{2}\right)\right)$.

Lemma 2.7 (Castelnuovo Riemann Roch theorem) [2, p 27]. Let $\pi: W_{g} \rightarrow W_{h}$ be an $m$ sheeted covering. Let $g_{N}^{R}$ be a linear series on $W_{g}$ where $N-R<g-m h$. Then any fiber of the map $\pi$ imposes at most $m-1$ linear conditions on $g_{N}^{R}$.

Lemma 2.8 [1]. Let $W_{g}$ admit a linear series $g_{n}^{1}$, without fixed points, and a half-canonical $g_{g-1}^{r}$, where $r \geq[n / 2]$. Then $g_{n}^{1}$ imposes at most $[n / 2]$ conditions on $g_{g-1}^{r}$.

\section{A generalization of the Castelnuovo-Severi inequality}

Suppose that $W_{g}$ admits two coverings

$$
\begin{aligned}
& \pi_{g h}: W_{g} \rightarrow W_{h} \text { in } m \text { sheets } \\
& \pi_{g k}: W_{g} \rightarrow W_{k} \text { in } n \text { sheets }
\end{aligned}
$$

where the two coverings admit no non-trivial common factorization. This array of coverings will be denoted $\operatorname{Iv}(g ; h, m ; k, n)$ where Iv stands for the letter "v" inverted to resemble the arrows in the diagram of these two coverings (with $W_{g}$ on top).

Suppose further that $W_{h}$ covers another Riemann surface, $W_{\ell}$ in $n$ sheets, and $W_{k}$ covers the same $W_{\ell}$ in $m$ sheets and the square diagram of covers commutes. ( $W_{g}$ on top and $W_{\ell}$ on the bottom.) We shall denote this array of coverings by $\mathrm{Sq}(g ; h, m ; k, n ; \ell)$. We will say that $W_{\ell}$ completes the Iv. ( $W_{\ell}$ is the unique completion since it corresponds to the intersection of two subfields of the full field of meromorphic functions on $W_{g}$.)

Let $W_{F}=W_{\ell}-P t_{F}$ where, as before, $P t_{F}$ is the set of points above which $\pi_{g \ell}$ is ramified. Let $A$ be the fundamental group of $W_{h}-\pi_{h \ell}^{-1}\left(P t_{F}\right) \quad\left(=W_{A}\right)$, of index $n$ in $F$. Let $B$ be the fundamental group of $W_{k}-\pi_{k \ell}^{-1}\left(P t_{F}\right)\left(=W_{B}\right)$ of index $m$ in $F$. Then $A \cap B$ is the fundamental group of $W_{g}-\pi_{g \ell}^{-1}\left(P t_{F}\right)$ $\left(=W_{A \cap B}\right)$ of index $m n$ in $F$. Also $F=A B$.

Now we wish to find an upper bound on $g$ given $m, n, h, k$, and $\ell$. This will give a weak generalization of the Castelnuovo-Severi inequality since we are assuming the $\mathrm{Iv}$ is completed. 
Since $F=A B=B A$ let

$$
\begin{array}{ll}
A b_{1}, A b_{2}, \ldots A b_{n} & b_{i} \in B \\
B a_{1}, B a_{2}, \ldots B a_{m} & a_{j} \in A
\end{array}
$$

be coset decompositions of $F$. Let $f(\in F)$ circle a point $t \in P t_{F}$. If $d_{1}, d_{2}, \ldots$ are the multiplicities of the points in $W_{h}$ above $t\left(\Sigma d_{i}=n\right)$ then the total ramification in $W_{h}$ above $t$ is $\Sigma\left(d_{i}-1\right)$. If $e_{1}, e_{2}, \ldots$ are the multiplicities of the points in $W_{k}$ above $t\left(\Sigma e_{j}=m\right)$ then the total ramification in $W_{k}$ above $t$ is $\Sigma\left(e_{j}-1\right)$. The total ramification of all points in $W_{g}$ above $t$ is $\Sigma \Sigma\left(d_{i}, e_{j}\right)\left\{\left[d_{i}, e_{j}\right]-1\right\}$.

Remark. If $d$ and $e$ are positive integers then $d e-d-e+(d, e) \geq 0$. Equality occurs if and only if $(d-1)(e-1)=0$.

LEMMA 3.1

$$
\Sigma \Sigma\left(d_{i}, e_{j}\right)\left\{\left[d_{i}, e_{j}\right]-1\right\} \leq m \Sigma\left(d_{i}-1\right)+n \Sigma\left(e_{j}-1\right)
$$

Equality occurs if and only if one of the two terms on the right hand side of $\left(^{*}\right)$ is zero.

\section{Proof}

$$
\begin{gathered}
m \Sigma\left(d_{i}-1\right)=\left(\Sigma e_{j}\right) \Sigma\left(d_{i}-1\right)=\Sigma \Sigma\left(e_{j} d_{i}-e_{j}\right) \\
n \Sigma\left(e_{j}-1\right)=\left(\Sigma d_{i}\right) \Sigma\left(e_{j}-1\right)=\Sigma \Sigma\left(d_{i} e_{j}-d_{i}\right)
\end{gathered}
$$

By the remark

$$
\left(e_{j} d_{i}-e_{j}\right)+\left(d_{i} e_{j}-d_{i}\right) \geq\left(d_{i}, e_{j}\right)\left\{\left[d_{i}, e_{j}\right]-1\right\}
$$

Summing on $i$ and $j$ proves the inequality. If we have equality in $(*)$ we have equality in all the inequalities $\left({ }_{i j}^{*}\right)$. If a term $\left(e_{j}-1\right) \neq 0$ then $\left(d_{i}-1\right)=0$ for all $i$.

Definition. Suppose for a point $t$ in $W_{\ell}$ for the square $\operatorname{Sq}(g ; h, m ; k, n ; \ell)$ at most only one of the two coverings of $W_{\ell}$ is ramified. The ramification at such a point will be said to be pure. If the ramification at every point of $W_{\ell}$ is pure then the covering $\pi_{g \ell}$ will be said to have separated ramification.

Theorem 3.2. Suppose we have a square $\operatorname{Sq}(g ; h, m ; k, n ; \ell)$. Then

$$
\begin{gathered}
2 g-2+m n(2 \ell-2) \leq m(2 h-2)+n(2 k-2) \\
(\text { or } g+m n \ell \leq m h+n k+(m-1)(n-1))
\end{gathered}
$$

Equality in (3.1) implies that the ramification for $\pi_{g \ell}$ is separated.

Proof. By the Riemann-Hurwitz formula (with obvious notation) 


$$
\begin{aligned}
2 g-2 & =m(2 h-2)+\operatorname{ram}(g, h) \\
2 g-2 & =n(2 k-2)+\operatorname{ram}(g, k) \\
2 g-2=m n(2 \ell-2)+ & \operatorname{ram}(g, \ell) \\
2 g-2+m n(2 \ell-2)+\operatorname{ram}(g, \ell)= & m(2 h-2)+n(2 k-2) \\
& +\operatorname{ram}(g, h)+\operatorname{ram}(g, k)
\end{aligned}
$$

But

$$
\operatorname{ram}(g, \ell)=\operatorname{ram}(g, h)+m \operatorname{ram}(h, \ell)=\operatorname{ram}(g, k)+n \operatorname{ram}(k, \ell)
$$

By Lemma 3.1

$$
\operatorname{ram}(g, \ell) \leq m \operatorname{ram}(h, \ell)+n \operatorname{ram}(k, \ell)
$$

Therefore

$$
\operatorname{ram}(g, k) \leq m \operatorname{ram}(h, \ell)
$$

And so: $\operatorname{ram}(g, \ell) \geq \operatorname{ram}(g, h)+\operatorname{ram}(g, k)$. Together with (3.2) this proves the inequality in the statement of the theorem.

If we have equality in (3.1) then we have equality in all the above inequalities, especially (3.3). Thus we have equality in $\left(^{*}\right)$ of Lemma 2.1 for each point of $P t_{F}$; that is, the ramification at each point of $P t_{F}$ is pure.

Corollary 3.3. Equality in (3.1) of Theorem 3.2 is equivalent to the following. (The $K$ 's refer to canonical series.)

1) $\operatorname{ram}(g, \ell)=\operatorname{ram}(g, h)+\operatorname{ram}(g, k)$

2) $\operatorname{ram}(g, h)=n \operatorname{ram}(k, \ell)$

3) $\operatorname{ram}(g, \ell)=n \operatorname{ram}(k, \ell)+m \operatorname{ram}(h, \ell)$

4) The ramification of $\pi_{g \ell}$ is separated.

$$
\text { 5) } K_{g}+\pi_{g \ell}^{-1}\left(K_{\ell}\right) \equiv \pi_{g h}^{-1}\left(K_{h}\right)+\pi_{g k}^{-1}\left(K_{k}\right)
$$

Proof. That 4) implies 3) together with the proof of Theorem 3.2 shows this corollary is true for the first four statements in the conclusion. We need only show that equality in (3.1) implies (3.4) since the converse is immediate. Let $B_{g h}$ denote the ramification divisor on $W_{g}$ for the covering $\pi_{g h}$ (with similar notation for other coverings.)

We show first that $B_{g h}=\pi_{g k}^{-1}\left(B_{k \ell}\right) . \quad \pi_{k \ell}$ is ramified only at points $t$ in $P t_{F}$ where the $f$ 's that circle the $t^{\prime} \mathrm{s}$ are in $A_{0}$, since the ramification of $\pi_{g \ell}$ is separated. These are the only points over which $\pi_{g h}$ is ramified. Above these points $\pi_{h \ell}$ and $\pi_{g k}$ are unramified. Thus $B_{g h}=\pi_{g k}^{-1}\left(B_{k \ell}\right)$.

Now $\quad K_{g} \equiv B_{g h}+\pi_{g h}^{-1}\left(K_{h}\right)$. Since $\pi_{g \ell}^{-1}\left(K_{\ell}\right)=\pi_{g k}^{-1}\left(\pi_{k \ell}^{-1}\left(K_{\ell}\right)\right) \quad$ and $\quad B_{g h}=$ $\pi_{g k}^{-1}\left(B_{k \ell}\right)$ we have

$$
K_{g}+\pi_{g \ell}^{-1}\left(K_{\ell}\right) \equiv \pi_{g k}^{-1}\left(B_{k \ell}+\pi_{k \ell}^{-1}\left(K_{\ell}\right)\right)+\pi_{g h}^{-1}\left(K_{h}\right) \equiv \pi_{g k}^{-1}\left(K_{k}\right)+\pi_{g h}^{-1}\left(K_{h}\right)
$$


COROLlary 3.4. Suppose for $\operatorname{Iv}(g ; h, m ; k, n)$ we have

$$
g+m n \ell_{0} \geq m h+n k+(m-1)(n-1)
$$

If the Iv is completed by $W_{\ell}$ then $\ell \leq \ell_{0}$.

Corollary 3.5. Assume we have equality in Theorem 3.2 and $l=0$. Then

$$
\operatorname{Mono}\left(W_{g} / W_{\ell}\right) \cong \operatorname{Mono}\left(W_{h} / W_{\ell}\right) \times \operatorname{Mono}\left(W_{k} / W_{\ell}\right)
$$

Proof. If $f$ circles a point in $P t_{F}$ then $f \in A_{0}$ or $f \in B_{0}$ but not both. Such $f$ 's generate $F$ so $F=A_{0} B_{0}$. Now

$$
\operatorname{Mono}\left(W_{g} / W_{\ell}\right) \cong F /\left(A_{0} \cap B_{0}\right) \cong A_{0} /\left(A_{0} \cap B_{0}\right) \times B_{0} /\left(A_{0} \cap B_{0}\right)
$$

But $\operatorname{Mono}\left(W_{h} / W_{\ell}\right) \cong F / A_{0} \cong B_{0} /\left(A_{0} \cap B_{0}\right) \ldots$ etc.

The following corollaries apply to Section 5 .

Definition. For a prime $p, p^{\alpha} \| n$, will mean that $p^{\alpha}$ divides $n$ but $p^{\alpha+1}$ does not. We say that $p^{\alpha}$ strictly divides $n$.

Corollary 3.6. Suppose we have a $\mathrm{Sq}(g ; h, p ; k, p ; \ell)$ where $p$ is an odd prime. Then $F=A_{0} B, p \|\left[F: A_{0}\right]$ and $p^{2} \|\left[F: A_{0} \cap B_{0}\right]$.

Proof. To show $F=A_{0} B$ it suffices to show that $A_{0} \not \subset B$ since $[F: B]=p$. If $A_{0} \subset B$ then $A_{0} \subset B_{0}$ and so $A_{0}=A_{0} \cap B_{0}$. But $F / A_{0}$ is isomorphic to a subgroup of $S_{p}$, and so $p \|\left[F: A_{0}\right]$. But $A_{0} \cap B_{0} \subset A \cap B$ and $p^{2}=[F: A \cap B]$. This contradiction shows that $F=A_{0} B$.

Now $F / A_{0} \cong B / A_{0} \cap B$. It follows that $\left[A_{0}: A_{0} \cap B\right]=p$. Also $\bigcap\left\{\left(A_{0} \cap B\right)^{g} \mid g \in A_{0}\right\}=A_{0} \cap B_{0}$ since $F=A_{0} B$. Consequently $p \|\left[A_{0}: A_{0} \cap B_{0}\right]$ and so $p^{2} \|\left[F: A_{0} \cap B_{0}\right]$.

COROllary 3.7. Suppose we have equality in Theorem 3.2 and $m=n=p$, an odd prime. Suppose there exists another subgroup $C \subset F$ of index $p$ containing $A \cap B$. Then the covering $W_{g} \rightarrow W_{C}^{*}$ is unramified.

Proof. $C \cap(A \cap B)=A \cap B=A \cap C=C \cap B$. Since $A, B$, and $C$ have index $p F=A C_{0}=A_{0} C=B C_{0}=B_{0} C$. We apply Lemma 2.1. Suppose $f \in F$ circles $t \in P t_{F}$. If $f \in A_{0}$ the covering $\left(W_{g}=\right) W_{A \cap C}^{*} \rightarrow W_{C}^{*}$ is unramified over $f$. If $f \in B_{0}$ the covering $\left(W_{g}=\right) W_{B \cap C}^{*} \rightarrow W_{C}^{*}$ is unramified over $f$. All points of $P t_{F}$ have been accounted for.

COROLlary 3.8. Suppose we have the hypotheses of Corollary 3.7 and $\ell=0$. Then $F /\left(A_{0} \cap B_{0}\right) \cong Z_{p} \times Z_{p}$.

Proof. Consider the finite group $F /\left(A_{0} \cap B_{0}\right)$; that is, we assume $A_{0} \cap B_{0}=$ $A_{0} \cap C_{0}=C_{0} \cap B_{0}=\langle e\rangle . \quad C_{0}$ is a normal subgroup of $F$, the direct product 
of $A_{0}$ and $B_{0}$, and $A_{0} \cap C_{0}=C_{0} \cap B_{0}=\langle e\rangle$. The Corollary now follows from Lemma 2.4 since $A_{0}, B_{0}$, and $C_{0}$, whose orders are divisible by $p$, are subgroups of $S_{p}$.

\section{Equality in the Castelnuovo-Severi inequality}

Definition. For a covering $\pi_{g h}: W_{g} \rightarrow W_{h}$ and a divisor $D$ on $W_{g}$ define

$$
\sigma_{g h}(D):=\pi_{g h}^{-1}\left(\pi_{g h}(D)\right) \text { a divisor of degree }\left(\operatorname{deg} \pi_{g h}\right)(\operatorname{deg} D)
$$

Suppose $W_{\ell}$ completes $\operatorname{Iv}(g ; h, m ; k, n)$. If $x \in W_{g}$ then $\sigma_{g \ell}(x)$ can be described in two ways: $\sigma_{g k}\left(\sigma_{g h}(x)\right)$ or $\sigma_{g h}\left(\sigma_{g k}(x)\right)$. For almost all $x$ in $W_{g} \sigma_{g \ell}(x)$ is a divisor of $m n$ distinct points. If $x$ is not in $\sigma_{g \ell}(y)$ then $\sigma_{g \ell}(x) \cap \sigma_{g \ell}(y)=\emptyset$. If $\ell=0$ then the $\sigma_{g l}(x)$ 's form a $g_{m n}^{1}$.

Lemma 4.1. Suppose we have an $\operatorname{Iv}(g ; h, m ; k, n)$, and for all but a finite number of $x$ on $W_{g} \sigma_{g k}\left(\sigma_{g h}(x)\right)=\sigma_{g h}\left(\sigma_{g k}(x)\right)$. Then there exists a $W_{\ell}$ $\left(=\left\{\sigma_{g k}\left(\sigma_{g h}(x)\right) \mid x \in W_{g}\right\}\right.$ which completes the Iv and $\sigma_{g \ell}(x)=\sigma_{g k}\left(\sigma_{g h}(x)\right)$.

Proof. $W_{q}^{\sim}:=\left\{x \in W_{g} \mid \operatorname{deg} \sigma_{g k}\left(\sigma_{g h}(x)\right)=m n\right.$ and $\left.\sigma_{g k}\left(\sigma_{g h}(x)\right)=\sigma_{g h}\left(\sigma_{g k}(x)\right)\right\}$. Then $W_{g}^{\sim}=W_{g}-\{$ a finite number of points $\}$. We first show that if $x_{1}, y \in W_{g}^{\sim}$ and $y \in \sigma_{g k}\left(\sigma_{g h}\left(x_{1}\right)\right)$ then $\sigma_{g k}\left(\sigma_{g h}\left(x_{1}\right)\right)=\sigma_{g k}\left(\sigma_{g h}(y)\right)$. Now let $\sigma_{g h}\left(x_{1}\right)=x_{1}+\cdots$ $+x_{m}$. There is an $i$ such that $y \in \sigma_{g k}\left(x_{i}\right)$. Thus $\sigma_{g k}\left(\sigma_{g h}(y)\right)=\sigma_{g h}\left(\sigma_{g k}(y)\right)=$ $\sigma_{g h}\left(\sigma_{g k}\left(x_{i}\right)\right)=\sigma_{g k}\left(\sigma_{g h}\left(x_{i}\right)\right)=\sigma_{g k}\left(\sigma_{g h}\left(x_{1}\right)\right)$.

Define $W_{\ell}^{\sim}=\left\{\sigma_{g k}\left(\sigma_{g h}(x)\right) \mid x \in W_{g}^{\sim}\right\}$. The maps from $W_{g}$ to $W_{h}$ and $W_{k}$, restricted to $W_{g}^{\sim}$ define topological maps from the latter to suitably punctured Riemann surfaces $W_{h}^{\sim}$ and $W_{k}^{\sim}$. Then $W_{\ell}^{\sim}$ completes the Iv formed by $W_{g}^{\sim}$, $W_{h}^{\sim}$, and $W_{k}^{\sim}$. Giving $W_{\ell}^{\sim}$ an analytic structure in the obvious manner and adding points to all the punctured Riemann surfaces completes the proof.

Definition. A Castelnuovo-Severi Iv, denoted $\operatorname{CSIv}(g ; h, m ; k, n)$, will be an Iv where

$$
g=m h+n k+(n-1)(m-1)
$$

It is difficult to believe that the following result is not somewhere in the literature of the last century.

THEOREM 4.2. Let $q$ be a prime in a $\operatorname{CSIv}(g ; h, m ; k, q)$. Assume $h>$ $(q-1)(q-2) / 2$. Then the CSIv admits a completion.

Proof. For each point $x \in W_{k} \pi_{g h}\left(\pi_{g k}^{-1}(x)\right)$ is a divisor of degree $q$ belonging to a single $g_{q}^{r}$ on $W_{h}$ by Lemma 2.6. Since $q$ is prime, $r>1$ implies that $W_{h}$ admits a plane model of degree $q$ and so $h \leq(q-1)(q-2) / 2$. Thus $r=1$.

Let $w_{1}$ be a point on $W_{g}$ so that the $m q$ points $\sigma_{g k}\left(\sigma_{g h}\left(w_{1}\right)\right)$ are unramified for $\pi_{g h}$ and $\pi_{g k} . \quad \sigma_{g h}\left(w_{1}\right)=w_{1}+\cdots+w_{m}$, and $\sigma_{g k}\left(\sigma_{g h}\left(w_{1}\right)\right)=\sigma_{g k}\left(w_{1}\right)+\cdots+$ 
$\sigma_{g k}\left(w_{m}\right)$. Each of the divisors $\pi_{g h}\left(\sigma_{g k}\left(w_{1}\right)\right), \ldots, \pi_{g h}\left(\sigma_{g k}\left(w_{m}\right)\right)$ belong to $g_{q}^{1}$ and all have the same point $\pi_{g h}\left(w_{1}\right)$ in common. Therefore, they are all the same divisor on $W_{h}$. It follows that $\pi_{g h}^{-1}\left(\pi_{g h}\left(\sigma_{g k}\left(w_{1}\right)\right)\right)=\sigma_{g k}\left(\sigma_{g h}\left(w_{1}\right)\right)$. The result follows from Lemma 4.1.

The following theorems are rather small steps in characterizing those CSIv's which have completions where the hypotheses of Theorem 4.2 do not hold. We shall be considering a $\operatorname{CSIv}(p q+1 ; 1, p ; 1, q)$ where $p$ and $q$ are odd primes. We believe that a completion for such an Iv is characterized by the existence on $W_{p q+1}$ of a complete half-canonical linear series $g_{p q}^{p+q-3}$ which is unique. We shall prove this only when $p$ or $q$ is 3 .

Let $g=p q+1$, let $\pi: W_{g} \rightarrow W_{1}$ be the $p$-sheeted covering, and let $\theta$ : $W_{g} \rightarrow T_{1}$ be the $q$-sheeted covering. Assume that $W_{g}$ admits a complete halfcanonical linear series $g_{p q}^{p+q-3}$. By the Castelnuovo Riemann-Roch theorem for $x$ on $W_{1} \pi^{-1}(x)$ imposes at most $p-1$ conditions on $g_{p q}^{p+q-3}$. The problem is to show that the words "at most" can be replaced by the word "precisely."

We first prove two lemmas from which our results follow.

Lemma 4.3. Suppose for a $\operatorname{CSIv}(p q+1 ; 1, p ; 1, q), W_{q}$ admits a halfcanonical $g_{p q}^{p+q-3+\varepsilon}, \varepsilon \geq 0$. Suppose there is a $g_{q}^{q-1}$ on $W_{1}$ so that $\left|\pi^{-1}\left(g_{q}^{q-1}\right)\right|=$ $g_{p q}^{p+q-3+\varepsilon}$. Then if $y_{0} \in T_{1}$ and $\theta^{-1}\left(y_{0}\right)$ consists of $q$ distinct points no two of which lie in a fiber of $\pi$ (there are a finite number of fibers omitted, by Lemma 2.5) then $\theta^{-1}\left(y_{0}\right)$ imposes precisely $q-1$ conditions on $g_{p q}^{p+q-3+\varepsilon}$.

Proof. Let $\theta^{-1}\left(y_{0}\right)=z_{1}, \ldots, z_{q}$. Suppose $z_{1}, \ldots, z_{t}$ impose $t$ independent conditions on $g_{p q}^{p+q-3+\varepsilon}$, so that if $D \in g_{p q}^{p+q-3+\varepsilon}$ and $D$ contains $z_{1}, \ldots, z_{t}$, then $D$ contains $z_{t+1}, \ldots, z_{q}$. We want to show that $t \geq q-1$. Assume otherwise. $\pi\left(z_{1}+\cdots+z_{q-1}\right)$ are $q-1$ points on $W_{1}$ which determine a divisor in $g_{q}^{q-1}$. Let that divisor be $\pi\left(z_{1}+\cdots+z_{q-1}\right)+x$. Then $\left|\pi\left(z_{q-1}\right)+x\right|=g_{2}^{1}$ on $W_{1}$. Choose a divisor $C$ in $g_{2}^{1}$ so that $\pi^{-1}(C) \cap\left\{z_{q-1}, z_{q}\right\}$ is empty. Then $\pi^{-1}\left(\pi\left(z_{1}+\cdots+\right.\right.$ $\left.\left.z_{q-2}\right)+C\right)$ is a divisor in $g_{p q}^{p+q-3+\varepsilon}$ containing $z_{1}+\cdots+z_{q-2}$ but not $z_{q-1}+z_{q}$. This contradiction proves the lemma.

Lemma 4.4. Suppose for a $\operatorname{CSIv}(p q+1 ; 1, p ; 1, q), W_{g}$ admits a halfcanonical $g_{p q}^{p+q-3+\varepsilon}, \varepsilon \geq 0$. Suppose there exists in $W_{1}$ an $x_{0}$ so that $\pi^{-1}\left(x_{0}\right)$ imposes precisely $p-1$ conditions on $g_{p q}^{p+q-3+\varepsilon}$. Then $\varepsilon=0$. If $D \in g_{p q}^{p+q-3}$ and $D$ contains $\pi^{-1}\left(x_{0}\right)$ then $D=\pi^{-1}\left(D_{q}\right)$ where $D_{q}$ is a divisor of degree $q$ on $W_{1}$.

Proof. If $x_{1} \in W_{1}, x_{1} \neq x_{0}$ then $\left|x_{1}+x_{0}\right|$ is a $g_{2}^{1}$ on $W_{1}$. On $W_{g} \pi^{-1}\left(x_{0}\right)+$ $\pi^{-1}\left(x_{1}\right)$, being a $g_{2 p}^{1}$, imposes $p$ conditions on $g_{p q}^{p+q-3+\varepsilon}$ (Lemma 2.8). Thus $\pi^{-1}\left(x_{1}\right)$ imposes one condition on $g_{p q}^{p+q-3+\varepsilon}-\pi^{-1}\left(x_{0}\right)\left(=g_{p q-p}^{q-2+\varepsilon}\right)$; that is, $g_{p q-p}^{q-2+\varepsilon}$ is composite being the lift of a $g_{q-1}^{q-2}$ on $W_{1}$, and so $\varepsilon=0 . \quad g_{p q}^{p+q-3}$ is now seen to be the completion of the lift of a $g_{q}^{q-1}$ on $W_{1}$. Thus the divisor in $g_{p q}^{p+q-3}$ containing $\pi^{-1}\left(x_{0}\right)$ is the lift of a divisor in $g_{q}^{q-1}$. 
TheOREM 4.5. Consider the $\operatorname{CSIv}(p q+1 ; 1, p ; 1, q)$, where $p$ and $q$ are odd primes and the Iv admits a completion. Then $W_{p q+1}$ admits a complete, simple half-canonical $g_{p q}^{p+q-3}$.

Proof. Since the completion $W_{\ell}$ has genus zero formula 3.4 in Corollary 3.3 insures that the $g_{1}^{1}$ on $W_{\ell}$ lifts to be half-canonical on $W_{g}$. $g_{1}^{1}$ lifted to $W_{1}$ completes to be $g_{q}^{q-1}$, and lifted to $T_{1}$ completes to a $g_{p}^{p-1}$. Lifted to $W_{g} g_{1}^{1}$ therefore has dimension at least $(p-1)+(q-1)-1$. Lemma 4.3 insures that there is a $y_{0}$ on $T_{1}$ so that $\theta^{-1}\left(y_{0}\right)$ imposes precisely $q-1$ conditions on the halfcanonical $g_{p q}^{p+q-3+\varepsilon}$, and Lemma 4.4 implies $\varepsilon=0$. Since $p$ and $q$ are prime it follows that $g_{p q}^{p+q-3}$ is simple.

THEOREM 4.6. For the $\operatorname{CSIv}(3 p+1 ; 1, p ; 1,3)$, suppose that $W_{3 p+1}$ admits a half-canonical $g_{3 p}^{p}$. Then the Iv admits a completion.

Proof. First we show that $g_{3 p}^{p}$ is simple. Suppose that it is composite. Since $g_{3 p}^{p}$ is not trigonal (by CSI), $W_{3 p+1}$ must cover a $W_{q}$ in two sheets. The non-fixed points of $g_{3 p}^{p}$ is the lift of a complete $g_{(3 p-f) / 2}^{p}$ on $W_{q}$ where $f$ is odd. Since the Clifford index is negative $q=(p-f) / 2$ and again this violates CSI.

Let $g=3 p+1$, let $\pi: W_{g} \rightarrow W_{1}$ be the $p$-sheeted covering, and let $\theta$ : $W_{g} \rightarrow T_{1}$ be the 3 -sheeted covering. Choose $y_{0} \in T_{1}$ so that $\theta^{-1}\left(y_{0}\right)$ is a divisor of three distinct points. Then $\theta^{-1}\left(y_{0}\right)$ imposes at most two conditions on $g_{3 p}^{p}$ by the Castelnuovo Riemann-Roch theorem. If $\theta^{-1}\left(y_{0}\right)$ imposed one condition $g_{3 p}^{p}$ would be composite, a contradiction. Thus $\theta^{-1}\left(y_{0}\right)$ imposes precisely two conditions. By Lemma 4.4 any divisor in $g_{3 p}^{p}$ containing $\theta^{-1}\left(y_{0}\right)$ is the lift of a divisor on $T_{1}$ of degree $p$. By Lemma 4.3, for a general choice of $x_{0}$ on $W_{1}$, $\pi^{-1}\left(x_{0}\right)$ imposes precisely $p-1$ conditions on $g_{3 p}^{p}$. By Lemma 4.4, again, any divisor in $g_{3 p}^{p}$ containing such a $\pi^{-1}\left(x_{0}\right)$ is the lift of a divisor of degree 3 on $W_{1}$. For a general point $z_{0}$ on $W_{g}, \pi^{-1}\left(\pi\left(z_{0}\right)\right)$ imposes $p-1$ conditions on $g_{3 p}^{p}$. A divisor in $g_{3 p}^{p}$ requires an one additional condition to contain $\theta^{-1}\left(\theta\left(z_{0}\right)\right)$. Thus it requires exactly $p$ conditions for a divisor in $g_{3 p}^{p}$ to contain $\pi^{-1}\left(\pi\left(z_{0}\right)\right)$ and $\theta^{-1}\left(\theta\left(z_{0}\right)\right)$, and so to be simultaneously lifted from divisors in $W_{1}$ and $T_{1}$. This gives a $g_{3 p}^{1}$ in $g_{3 p}^{p}$ which completes the Iv.

In this case the half-canonical $g_{3 p}^{p}$ is the unique linear series on $W_{3 p+1}$ of dimension $p$ and degree $3 p$.

\section{Several Castelnuovo-Severi coverings}

Definition. For $p$ an odd prime, let $g(p)=p^{3}-2 p^{2}+1$ and let $h(p)=$ $(p-1)(p-2) / 2$.

In this section we shall always assume that all the $\operatorname{CSIv}(g(p) ; h(p), p ; h(p)$, $p)$ 's which occur have completions. This generalizes the case $p=3$, where we have given conditions which insure that completions occur [3]. 
Consider the fundamental group, $F$, of a punctured Riemann surface $W_{F}$, together with three subgroups $A, B$, and $C$, all of index $p$ in $F$. Then we have three additional subgroups $A \cap B, A \cap C$, and $B \cap C$, which we assume all have index $p^{2}$ in $F$, and we have $A \cap B \cap C$ which we assume has index $p^{3}$ in $F$. Then $W_{F}$ is covered by seven Riemann surfaces $W_{H}$ with fundamental group $H$, where $H$ is any one of the seven proper subgroups of $F$ mentioned above. In this array of eight Riemann surfaces $W_{F}$ will be called the $0^{\text {th }}$ or bottom level; $W_{H}, H=A, B$, or $C$ will be called the first level; $W_{H}, H=A \cap B, A \cap C$, or $B \cap C$ will be called the second level, and $W_{A \cap B \cap C}$ will be called the third or top level. The Riemann surfaces on the same level (ith) will always be assumed to have the same genus $\left(h_{i}\right)$. This array of eight Riemann surfaces will be denoted $\mathrm{Cu}\left(h_{3}, h_{2}, h_{1}, h_{0}\right)$. This array can be visualized as a cube standing on one of its vertices, the vertices standing for the Riemann surfaces and the edges pointing downward standing for the covering maps. The cubes of interest will be $\mathrm{Cu}(g(p), h(p), 0,0)$ which we will abbreviate by $\mathrm{Cu}(p)$.

We shall also consider the possibility of four subgroups of $F, A, B, C, D$ all of index $p$ in $F$, the corresponding six subgroups, $A \cap B, A \cap C, \ldots, C \cap D$ all of index $p^{2}$ in $F$, the four subgroups $A \cap B \cap C, \ldots, B \cap C \cap D$ all of index $p^{3}$ in $F$ and $A \cap B \cap C \cap D$ of index $p^{4}$ in $F$. There are five levels for the corresponding Riemann surfaces, and if $h_{i}$ is the genus of all Riemann surfaces at the $i$ th level, this array of 16 Riemann surfaces will be denoted $\mathrm{HyCu}\left(h_{4}, h_{3}, h_{2}, h_{1}, h_{0}\right)$. This can perhaps be visualized as a hypercube standing "vertically" on one of its vertices, with vertices and downward pointing edges standing for Riemann surfaces and covering maps as in a cube. Our purpose will be to show that a $\mathrm{HyCu}(g(p), h(p), 0,0,0)$, denoted $\mathrm{HyCu}(p)$, does not exist.

Then we will show that a $W_{g(p)}$ covering four different $W_{h(p)}$ 's, each in $p$ sheets with all Iv's admitting completions, must admit a group of automorphisms isomorphic to the elementary abelian group of order $p^{3}$, with four subgroups of order $p$ giving rise to the four coverings $W_{g(p)} \rightarrow W_{h(p)}$. Except for $p=3$, we will avoid the problem of completing the $\operatorname{Iv}(g(p) ; h(p), p ; h(p), p)$. If we have such an Iv completed to a square we will denote it $\operatorname{Sq}(g(p), h(p), 0)$ or more simply $\mathrm{Sq}(p)$.

Lemma 5.1. Suppose $W_{g}$ admits three coverings $W_{g} \rightarrow W_{h}, W_{g} \rightarrow W_{k}$, $W_{g} \rightarrow W_{\ell}$, in $p, q$, and $r$ sheets respectively (all primes). Suppose the three Iv's $(\operatorname{Iv}(g ; h, p ; k, q)$ etc.) all have completions. Then the array of seven Riemann surfaces with three coverings and three squares can be completed to a cube of eight Riemann surfaces.

Proof. For almost all $w \in W_{g}$ we want a divisor of degree $p q r$ containing $w$, equal to $\sigma_{g \tau(h)}\left(\sigma_{g \tau(k)}\left(\sigma_{g \tau(\ell)}(w)\right)\right)$ for any permutation $\tau$ of the letters $h, k, \ell$. Now $\sigma_{g h}\left(\sigma_{g k}\left(\sigma_{g \ell}(w)\right)\right)=\sigma_{g h}\left(\sigma_{g \ell}\left(\sigma_{g k}(w)\right)\right)$. But for each point $v \in \sigma_{g \ell}(w) \sigma_{g h}\left(\sigma_{g k}(v)\right)=$ $\sigma_{g k}\left(\sigma_{g h}(v)\right)$. Therefore $\sigma_{g h}\left(\sigma_{g k}\left(\sigma_{g \ell}(w)\right)\right)=\sigma_{g k}\left(\sigma_{g h}\left(\sigma_{g l}(w)\right)\right)$. Therefore, any permutation of the $\sigma$-symbols is possible. The proof is now completed as in Lemma 4.1. 
An entirely analogous proof gives:

Lemma 5.2. Suppose $W_{g}$ admits 4 coverings $W_{g} \rightarrow W_{g^{\prime}} g^{\prime}=h, k, \ell, m$, all of prime degree. Suppose all 6 Iv's have completions. Then the array of 15 Riemann aurfaces (4 coverings, 6 squares, and 4 cubes) can be completed to a hypercube of 16 Riemann surfaces.

Let us summarize the results of Theorem 3.2 in the case of a $\mathrm{Sq}(p)$. Let $F$ be the fundamental group of the punctured $\mathbf{P}^{1}, W_{F}$. Let $A$ and $B$ be the subgroups of $F$ which are the fundamental groups for the two punctured $W_{h(p)}$. Then $A \cap B$ is the fundamental group of the punctured $W_{g(p)}$. If $t \in P t_{F}$ and $f(\in F)$ circles $t$ then $f \in A_{0} \cup B_{0}$ since the ramification of $W_{g(p)} \rightarrow W_{F}^{*}\left(=\mathbf{P}^{1}\right)$ is separated. Therefore, $F=A_{0} B_{0}$ since $A_{0}$ and $B_{0}$ are normal in $F$. $W_{F}^{*}=\mathbf{P}^{1}$. $\operatorname{Mono}\left(W_{g(p)} / W_{F}^{*}\right) \cong \operatorname{Mono}\left(W_{A}^{*} / W_{F}^{*}\right) \times \operatorname{Mono}\left(W_{B}^{*} / W_{F}^{*}\right)$.

Now we examine the cube $\mathrm{Cu}(p)$. Assume that the corresponding array of subgroups of $F$ arise from subgroups $A, B$, and $C$ (all of index $p$ in $F$ ). In this case $W_{A}^{*}, W_{B}^{*}$, and $W_{C}^{*}$ are all of genus zero.

Corollary 3.6 says that $F=A B_{0}$. We also obtain true statements by replacing $A$ and $B$ by any two of the letters $A, B$, or $C$. We shall have further results stated in terms of a set of subgroups of $F$ where the result holds for the statement modified by permuting the names of the subgroups. We shall use such statements by referring to the original statement without further comment.

Lemma 5.3. In the cube $\mathrm{Cu}(p)$, suppose $t \in P t_{F}$ and $f(\in F)$ circles $t$. If $f \in A_{o}$, then $f \in B_{0} \cup C_{0}$.

Proof. Let $A x_{1}, \ldots, A x_{p}$ be a coset decomposition of $F$. The points above $t$ in $W_{A}^{*}$ are circled by the $p$ curves $x_{i} f x_{i}^{-1}$. Since $W_{A \cap B \cap C}^{*} \rightarrow W_{A}^{*}$ has separated rami-fication, $x_{i} f x_{i}^{-1} \in \bigcap\left\{(A \cap B)^{a} \mid a \in A\right)$ or $x_{i} f x_{i}^{-1} \in \bigcap\left\{(A \cap C)^{a} \mid a \in A\right)$. By Lemma 2.2, $f \in B_{0}$ or $f \in C_{0}$.

Lemma 5.4. For a $\mathrm{Cu}(p)$ let $f(\in F)$ circle a point $t$ in $P f_{F}$. If $f \notin A_{0} \cup$ $B_{0} \cup C_{0}$ then $f^{p} \in A_{0} \cap B_{0} \cap C_{0}$.

Proof. Suppose the multiplicities of the covering maps over $t$ are as follows:

With respect to $W_{A}^{*} \rightarrow W_{F}^{*}$ they are $\alpha_{1}, \alpha_{2}, \ldots, \Sigma \alpha_{i}=p$

With respect to $W_{B}^{*} \rightarrow W_{F}^{*}$ they are $\beta_{1}, \beta_{2}, \ldots, \Sigma \beta_{j}=p$

With respect to $W_{C}^{*} \rightarrow W_{F}^{*}$ they are $\gamma_{1}, \gamma_{2}, \ldots, \Sigma \gamma_{k}=p$

We wish to show that $\alpha_{1}=\beta_{1}=\gamma_{1}=p$. Suppose otherwise. Assume $\alpha^{\prime}$, one of the $\alpha^{\prime}$ s, is the smallest of all the $\alpha^{\prime}$ s, $\beta$ 's, and $\gamma^{\prime}$ s, $1 \leq \alpha^{\prime}<p$. We claim that there is a $\beta$, call it $\beta^{\prime}$ so that $\beta^{\prime}>\alpha^{\prime}$. If not, all the $\beta^{\prime}$ s equal $\alpha^{\prime}$, and so $\alpha^{\prime}$ divides $p$. Thus $\alpha^{\prime}=1$ and so $f \in B_{0}$. Contradiction.

Similarly there is a $\gamma^{\prime}$ so that $\gamma^{\prime}>\alpha^{\prime}$. 
Let $B v_{1}, \ldots, B v_{p}$ be a coset decomposition of $F$. There is a $v$, call it $v_{j}$, so that $B v_{j} f^{\beta^{\prime}}=B v_{j}$ and $\beta^{\prime}$ is the smallest positive integer with this property. Therefore $B v_{j} f^{\alpha^{\prime}} \neq B v_{j}$, so $f^{\alpha^{\prime}} \notin B_{0}$. Similarly, $f^{\alpha^{\prime}} \notin C_{0}$.

But there is an $x$ so that $A x f^{\alpha^{\prime}}=A x$ or $x f^{\alpha^{\prime}} x^{-1} \in A$. Since $W_{A \cap B \cap C}^{*} \rightarrow W_{A}^{*}$ has separated ramification $x f^{\alpha^{\prime}} x^{-1} \in\left(A \cap B_{0}\right) \cup\left(A \cap C_{0}\right)$ or $f^{\alpha^{\prime}} \in B_{0} \cup C_{0}$. This contradiction proves the lemma.

We summarize the discussion so far in a theorem.

THEOREM 5.5. Let the cube $\mathrm{Cu}(p)$ correspond to the fundamental group $F$ of $W_{F}$. Let $F$ have subgroups $A, B$, and $C$ as before. For $t \in P t_{F}$ let $f \in F$ circle t. Then either $f \in\left(A_{0} \cap B_{0}\right) \cup\left(A_{0} \cap C_{0}\right) \cup\left(B_{0} \cap C_{0}\right)$ or $f^{p} \in A_{0} \cap B_{0} \cap C_{0}$. That is, for the coverings $W_{H}^{*} \rightarrow W_{F}^{*}, H=A, B, C$, above t, either two of the three are unramified or all are ramified with multiplicity $p$. Above one and only one point $t \in P t_{F}$ does the latter hold.

Proof. Only the last sentence needs verification. Since the ramification is not separated from level two to level zero, there must be at least one such point. If there were two such points all the ramification between Riemann surfaces of level one and level zero would be accounted for since these Riemann surfaces are all Riemann spheres. This is a contradiction.

Corollary 5.6. Continue the hypotheses of Theorem 5.5. Then $F=$ $A_{0}\left(B_{0} \cap C_{0}\right)$.

$$
\operatorname{Mono}\left(W_{A \cap B \cap C} / W_{F}\right) \cong \operatorname{Mono}\left(W_{A} / W_{F}\right) \times \operatorname{Mono}\left(W_{B} / W_{F}\right) \times \operatorname{Mono}\left(W_{C} / W_{F}\right) .
$$

Proof. That $F=A\left(B_{0} \cap C_{0}\right)$ is shown by a proof analogous to that of Corollary 3.6. Theorem 5.5 implies that $F$ is generated by elements in $A_{0} \cap B_{0}$, $A_{0} \cap C_{0}$, and $B_{0} \cap C_{0}$. For notational convenience assume that $A_{0} \cap B_{0} \cap C_{0}=$ $\langle e\rangle$, that is $F$ is now isomorphic to $\operatorname{Mono}\left(W_{A \cap B \cap C} / W_{F}\right)$ and $F=\left(A_{0} \cap B_{0}\right) \times$ $\left(A_{0} \cap C_{0}\right) \times\left(B_{0} \cap C_{0}\right) . \quad$ Now it is seen that $F=A_{0}\left(B_{0} \cap C_{0}\right) . \quad \operatorname{Mono}\left(W_{A} / W_{F}\right) \cong$ $F / A_{0} \cong\left(B_{0} \cap C_{0}\right)$ etc.

TheOrem 5.7. A $\mathrm{HyCu}(p)$ does not exist.

Proof. Suppose such a hypercube does exist. Let the fundamental groups at the various levels be $F, A, B, C, D, A \cap B, \ldots, A \cap B \cap C, \ldots, A \cap B \cap C \cap D$. For $t \in P t_{F}$ let $f \in F$ circle $t$. We show

1) If $f \in A_{0}$ then $f \in\left(B_{0} \cap C_{0}\right) \cup\left(B_{0} \cap D_{0}\right) \cup\left(C_{0} \cap D_{0}\right)$.

2) If $f \notin A_{0} \cup B_{0} \cup C_{0} \cup D_{0}$ then $f^{p} \in A_{0} \cap B_{0} \cap C_{0} \cap D_{0}$.

1) As in Lemma 5.3, suppose $f \in A_{0}$. If $A x_{1}, \ldots, A x_{p}$ is a coset decomposition for $F$ then $x_{i} f x_{i}^{-1} \in A_{0}$ for all $i$. The covering $W_{A \cap B \cap C \cap D}^{*} \rightarrow W_{A}^{*}$ is a $\mathrm{Cu}(p)$, so either $x_{i} f x_{i}^{-1} \in\left(A_{0} \cap\left(B_{0} \cap C_{0}\right)\right) \cup\left(A_{0} \cap\left(B_{0} \cap D_{0}\right)\right) \cup\left(A_{0} \cap\left(C_{0} \cap D_{0}\right)\right)$ or $x_{i} f^{p} x_{i}^{-1} \in A_{0} \cap B_{0} \cap C_{0} \cap D_{0}$. But this latter alternative would hold for all paths $x_{i} f x_{i}^{-1}$ circling the in $P t_{A}$ above $t$. Since there is at most only one point in $P t_{A}$ above $t$ with this property (Theorem 5.5) the second alternative is ruled out. 
2) If $f \notin A_{0} \cup B_{0} \cup C_{0} \cup D_{0}$ then there are four coverings $W_{H}^{*} \rightarrow W_{F}^{*}, H=$ $A, B, C, D$, and all are ramified over $t$. Using the arguments of Lemma 5.4 (multiplicities over $t$ with respect to $W_{D}^{*} \rightarrow W_{F}^{*}$ are $\delta_{1}, \delta_{2}, \ldots, \Sigma \delta_{\ell}=p$ ) let $\alpha^{\prime}$, the smallest of all the $\alpha$ 's, $\beta$ 's, $\gamma^{\prime}$ s, $\delta$ 's. We can then find $\beta^{\prime}, \gamma^{\prime}, \delta^{\prime}$, so that $\beta^{\prime}>\alpha^{\prime}$, $\gamma^{\prime}>\alpha^{\prime}, \delta^{\prime}>\alpha^{\prime}$. As in Lemma 5.4 we conclude that $f^{\alpha^{\prime}} \notin B_{0} \cup C_{0} \cup D_{0}$. There is a $x_{1}$ so that $A x_{1} f^{\alpha^{\prime}}=A x_{1}$ or $x_{1} f^{\alpha^{\prime}} x_{1}^{-1} \in A$. Let $x_{1} f^{\alpha^{\prime}} x_{1}^{-1}$ circle $a \in P t_{A}$. Since $W_{A \cap B \cap C \cap D}^{*} \rightarrow W_{A}^{*}$ is a $\mathrm{Cu}(p)$ the ramification of $W_{A \cap H}^{*} \rightarrow W_{A}^{*}(H=B, C$, $D)$ is not pure above $a$. We conclude that $\left(x_{1} f^{\alpha^{\prime}} x_{1}^{-1}\right)^{p} \in A \cap B_{0} \cap C_{0} \cap D_{0}$.

Consider the square with $W_{A \cap B}^{*}$ at the top and $W_{F}^{*}$ at the bottom. $a$ is the point in $P t_{A}$ above $t$ where the multiplicity of $\pi_{A, F}$ is $\alpha^{\prime}$. Let $b$ be the point in $P t_{B}$ above $t$ where the multiplicity of $\pi_{B, F}$ is $\beta^{\prime}$. If $c$ is a point in $P t_{A \cap B}$ above $t$ that maps onto $a$ and $b$, the last paragraph shows that the multiplicity of $\pi_{A \cap B, F}$ at $c$ is $\alpha^{\prime} p$. But by the discussion preceding Lemma 2.1 this multiplicity is also $\left[\alpha^{\prime}, \beta^{\prime}\right]$. Since $\alpha^{\prime}<\beta^{\prime} \leq p$, it follows that $\beta^{\prime}=p$. Similarly $\gamma^{\prime}=\delta^{\prime}=p$.

Since $\alpha^{\prime}<p$, there is another point $e \in P t_{A}$ over $t$. If $\varepsilon$ is the multiplicity of $\pi_{A, F}$ at $e$ then $\varepsilon<p$. Since we know that $\beta^{\prime}=\gamma^{\prime}=\delta^{\prime}=p$ we have $\beta^{\prime}=\gamma^{\prime}=$ $\delta^{\prime}=p>\varepsilon$. The same argument as above now shows that there is a $x_{2}$ so that $x_{2} f^{\varepsilon} x_{2}^{-1} \in A$ and $\left(x_{2} f^{\varepsilon} x_{2}^{-1}\right)^{p} \in A \cap B_{0} \cap C_{0} \cap D_{0}$. $W_{A}^{*}$ has two points, $a$ and $e$, over which each of the three coverings $W_{A \cap H}^{*} \rightarrow W_{A}^{*}, H=B, C, D$ has ramification $p$. Since this contradicts Theorem 5.5, we conclude that $\alpha^{\prime}=p$, and so $f^{p} \in A_{0} \cap B_{0} \cap C_{0} \cap D_{0}$.

We now conclude the proof. As before there is one and only one point in $\mathrm{Pt}_{F}$ where alternative 2) holds. This implies that the ramification for any square between levels one and three is separated. Since this is not the case, this contradiction shows that a $\mathrm{HyCu}(p)$ does not exist.

For Theorem 5.7 a proof similar to the one presented appears necessary for there exists a $\mathrm{HyCu}\left(\left(3 p^{4}-5 p^{3}+2\right) / 2, g(p), h(p), 0,0\right)$ where items 1) and 2) in the proof are satisfied.

THEOREM 5.8. Let $W_{g(p)}$ cover four different $W_{h(p)}$ 's, each in $p$ sheets, so that each of the six Iv's admit completions. Then $W_{g(p)}$ admits an automorphism group, $G$, iso-morphic to $Z_{p} \times Z_{p} \times Z_{p}$, and the four $W_{h(p)}$ 's are $W_{g(p)}$ modulo four of the $Z_{p}$ 's in $G$.

Proof. Three of the coverings $W_{g(p)} \rightarrow W_{h(p)}$ give rise to a $\mathrm{Cu}(p)$ since three of these coverings cannot be in a square (Corollary 3.7). The fourth such covering must occur within this cube (Theorem 5.7); that is, there are four cubes within one cube. Let $A, B, C, D$ be the four subgroups of $F$ of index $p$, as before. Then $A \cap B \cap C=A \cap B \cap D=\cdots=A \cap B \cap C \cap D$. For notational convenience let $A_{0} \cap B_{0} \cap C_{0} \cap D_{0}=\langle e\rangle$. By Theorem $5.6 F$ is equal to a product of three different groups $H_{0} \cap L_{0}$ where $H_{0} \neq L_{0}$ and $\{H, L\}$ is any of the three pairs in a subset of $\{A, B, C, D\}$ of order three. Then $\left(A_{0} \cap B_{0}\right) \times$ $\left(A_{0} \cap C_{0}\right) \times\left(B_{0} \cap C_{0}\right)=\left(A_{0} \cap B_{0}\right) \times\left(A_{0} \cap D_{0}\right) \times\left(B_{0} \cap D_{0}\right)$, and so $\left(A_{0} \cap D_{0}\right)$ is a normal subgroup of $\left(A_{0} \cap C_{0}\right) \times\left(B_{0} \cap C_{0}\right)$ intersecting each factor in the direct 
product in $\langle e\rangle . \quad A_{0} \cap D_{0}$ is isomorphic to $\operatorname{Mono}\left(W_{B} / W_{F}\right)$ and so is isomorphic to a subgroup of $S_{p}$, a subgroup whose order is divisible by $p$. By Lemma 2.4 $A_{0} \cap D_{0}$ is isomorphic to $Z_{p}$. By similar arguments we complete the proof of the theorem.

\section{Examples}

Let $W_{F}=\mathbf{P}^{1}-\left\{t_{1}, t_{2}, \ldots, t_{s}\right\}$, the $s$-fold punctured Riemann sphere. Let $f_{1}, f_{2}, \ldots, f_{s}$, be paths in $W_{F}$ which circle the $t$ 's so that $F=\left\langle f_{1}, f_{2}, \ldots, f_{s}\right|$ $\left.f_{1} f_{2} \cdots f_{s}=e\right\rangle$. Let $G$ be a finite group generated by $a_{1}, a_{2}, \ldots, a_{s-1}$. Let $\mu$ : $F \rightarrow G$ be defined by $\mu\left(f_{i}\right)=a_{i}$ for $i=1,2, \ldots, s-1$, and $\mu\left(f_{s}\right)=\left(a_{1} a_{2} \ldots\right.$ $\left.a_{s-1}\right)^{-1}$. $\quad \mu$ extends to a homomorphism from $F$ onto $G$. Let $H$ be a subgroup of $G$ of index $n$ such that $H_{0}=\langle e\rangle$. Let $A=\mu^{-1}(H)$. Then $W_{A}$ is an $n$-sheeted covering of $W_{F}$ corresponding to $H$ and $F / A_{0} \cong G$. Suppose $G$ has order $m$. $W_{A_{0}}^{*}$ is the Galois closure for the covering $W_{A}^{*} \rightarrow W_{F}^{*}$.

Notation. $W_{m}\left(f_{1}, f_{2}, \ldots, f_{s}\right)$ will denote $W_{A_{0}}, W_{A}$ will be denoted by $W_{n}\left(f_{1}, f_{2}, \ldots, f_{s}\right)$.

In the following we will consider only cyclic groups, $Z_{p}$, and dihedral groups, $D_{p}$, as subgroups of $S_{p}$, in order to build our direct products, $G$. Any other subgroup of $S_{p}$ containing $Z_{p}$, such as $A_{p}$ or $S_{p}$, would do, although the computations would be more complicated. Dihedral groups will be denoted $\langle a, \alpha\rangle$, $\langle b, \beta\rangle,\langle c, \gamma\rangle$ where $a^{2}=b^{2}=c^{2}=\alpha^{p}=\beta^{p}=\gamma^{p}=e$, and cyclic groups will be denoted simply by $\langle\alpha\rangle,\langle\beta\rangle,\langle\gamma\rangle$. Thus a $2 p$-sheeted dihedral Galois covering of $\mathbf{P}^{1}$ is denoted $W_{2 p}\left(a, a \alpha, \alpha^{-1}\right)$, and the $p$-sheeted covering of $\mathbf{P}^{1}, W_{2 p}\left(a, a \alpha, \alpha^{-1}\right) /$ $\langle a\rangle$, is denoted by $W_{p}\left(a, a \alpha, \alpha^{-1}\right)$. There are, of course, many such $p$-sheeted coverings corresponding to the conjugates of $\langle a\rangle$ in $D_{p}$.

An example of a $\mathrm{Sq}\left((p-1)^{2} ; 0, p ; 0, p ; 0\right)$ is given by $W_{p^{2}}(a, a, a \alpha, a \alpha, b, b, b \beta$, $b \beta)$ where $G=D_{p} \times D_{p}$. Examples of $\mathrm{Cu}(g(p), h(p), 0,0)$ are:

$$
\begin{array}{lll}
\text { (i) } & W_{p^{3}}\left(a, a \alpha, b, b \beta, c, c \gamma,(\alpha \beta \gamma)^{-1}\right) & G=D_{p} \times D_{p} \times D_{p} \\
\text { (ii) } & W_{p^{3}}\left(a, a \alpha, b, b \beta, \gamma,(\alpha \beta \gamma)^{-1}\right) & G=D_{p} \times D_{p} \times Z_{p} \\
\text { (iii) } & W_{p^{3}}\left(a, a \alpha, \beta, \gamma,(\alpha \beta \gamma)^{-1}\right) & G=D_{p} \times Z_{p} \times Z_{p} \\
\text { (iv) } & W_{p^{3}}\left(\alpha, \beta, \gamma,(\alpha \beta \gamma)^{-1}\right) & G=Z_{p} \times Z_{p} \times Z_{p}
\end{array}
$$

As an example we will work out the case (ii). Let $\lambda$ be the genus of $W_{4 p^{3}}\left(a, a \alpha, b, b \beta, \gamma,(\alpha \beta \gamma)^{-1}\right)$. Then

$$
2 \lambda-2=-8 p^{3}+4\left(4 p^{3} / 2\right)+2\left(4 p^{2}(p-1)\right)
$$

$W_{\lambda}$ is a 4-sheeted Galois covering of $W_{p^{3}}\left(a, a \alpha, b, b \beta, \gamma,(\alpha \beta \gamma)^{-1}\right)$ of genus $g$. $W_{g}=W_{\lambda} /\langle a, b\rangle$ where $\langle a, b\rangle \cong Z_{2} \times Z_{2}$. Since an involution in $D_{p}$ has $p$ conjugates, an involution in $\langle a, \alpha\rangle$ will have $2\left(4 p^{3} / 2\right) / p\left(=4 p^{2}\right)$ fixed points. $a b$ 
is an involution without fixed points. Thus the total ramification for $W_{\lambda} \rightarrow W_{g}$ is $2\left(4 p^{2}\right)$ and so

$$
2 \lambda-2=4(2 g-2)+8 p^{2}, \quad \text { and so } g=g(p) .
$$

To find the genus of the Riemann surface covered by $W_{g}$ in $p$ sheets, call it $W_{h}$, we could either consider $W_{2 p^{2}}\left(b, b \beta, \gamma,(\beta \gamma)^{-1}\right)$ and proceed as above, or consider $W_{\lambda} /\langle a, \alpha, b\rangle$. In the latter case $\langle a, \alpha, b\rangle$ has $p+1$ involutions with ramification $2 p^{2}$ for each. All the other subgroups are unramified or contain one of the ramified involutions. Therefore,

$$
2 \lambda-2=4 p(2 h-2)+(p+1) 4 p^{2}, \quad \text { or } \quad h=h(p) .
$$

$W_{\lambda} /\langle a, b, \gamma\rangle$ also has genus $h(p)$ since the ramification accounted for in $\langle a, b, \gamma\rangle$ is $2\left(4 p^{2}\right)+4 p^{2}(p-1)$.

In case (iv) we have four coverings $W_{g(p)} \rightarrow W_{h(p)}$, one for each of the four punctures in $\mathbf{P}^{1}$. The kernel of $\mu: F \rightarrow Z_{p} \times Z_{p} \times Z_{p}$ is a characteristic subgroup of $F$, so any fractional linear transformation (FLT) of $\mathbf{P}^{1}$ that permutes the four points lifts to $W_{g(p)}$. There is always a $Z_{2} \times Z_{2}$ of such FLT's, but by special arrangements of the four points we can have a dihedral group $D_{4}$ (order 8) or an alternating group $A_{4}$ (order 12) permuting the four points. Thus $W_{g(p)}$ always admits an automorphism group of order $4 p^{3}$, but can also admit groups of order $8 p^{3}$ and $12 p^{3}$.

\section{Genus ten}

On a Riemann surface $W_{10}$ a quartet is a set of four complete half-canonical linear series: $g_{9}^{2}, h_{9}^{2}, k_{9}^{2}, \ell_{9}^{3}$, whose sum is bicanonical, and where $\ell_{9}^{3}$ is the unique linear series on $W_{10}$ of dimension 3 and degree 9. By Riemann's solution to the Jacobi inversion problem [5] this is equivalent to the vanishing of the theta function for $W_{10}$ at four half periods, whose sum is zero, to orders $3,3,3,4$, where the last half-period is the only point on the Jacobian where the theta function vanishes to order 4 or more.

In [3] it was shown that the existence of a quartet on a $W_{10}$ is equivalent to the existence of a full three-sheeted covering $W_{10} \rightarrow W_{1}$ ("full" means that $K_{10}$ is the completion of the lift of a $g_{6}^{5}$ on $W_{1}$ ). Unfortunately, the methods do not appear to distinguish between cyclic and dihedral coverings.

The existence of two quartets, (necessarily with the same $\ell_{9}^{3}$ ) gives a CSIv which admits a completion by Theorem 4.6. The existence of three quartets leads to a $\mathrm{Cu}(10,1,0,0)$. The existence of four quartets leads to the existence of an elementary abelian group of order 27 on $W_{10}$, four subgroups of order three giving rise to the four coverings $W_{10} \rightarrow W_{1}$ (Theorem 5.8).

Conversely, the existence of such a group of automorphisms on $W_{10}$ implies the existence of six $\operatorname{CSIv}(10 ; 1,3 ; 1,3)$ 's all of which have completions. The proof of Theorem 4.5 shows that all the coverings $W_{10} \rightarrow W_{1}$ are full. Thus $W_{10}$ admits four quartets. 
THEOREM 7. For a Riemann surface of genus $10, W_{10}$, the following two statements are equivalent.

(1) $W_{10}$ admits an elementary abelian group of order 27 . Four cyclic subgroups give rise to quotients of genus one, and the remaining 9 cyclic subgroups are fixed point free.

(2) $W_{10}$ admits 4 quartets.

Remarks. 1) The $W_{10}$ in Theorem 7 can be simply described as a $W_{10}$ admitting an elementary abelian group, $G$, of order 27 so that the genus of $W_{10} / G$ is zero.

2) By the remarks at the end of Section 6 we have the following. Any such $W_{10}$ admits an automorphism group of order 4.27. There are such $W_{10}$ 's admitting automorphism groups of order 8.27 and 12.27 .

3) The curve $x^{6}+y^{6}+z^{6}=0$ is not such a Riemann surface since the 3-Sylow subgroup is not abelian.

\section{Coverings of the Riemann sphere}

In this section we consider $\operatorname{CSIv}(g ; 0, p ; 0, q)$ 's where $g=(p-1)(q-1)$, and $p$ and $q$ need not be prime.

We first do some naïve dimension counting. A generic $W_{g}$ on the top of such an Iv admits a plane model as a plane curve of degree $p+q$ with two ordinary singularities of multiplicities $p$ and $q$. The dimension of such a family of plane curves is $[(p+q+3)(p+q)-p(p+1)-q(q+1)] / 2-4=p q+p+$ $q-4$. The two singularities arise from picking a divisor from each of the two distinguished linear series on the Riemann surface, so the dimension in moduli space for genus $p q+1$ is $p q+p+q-6$.

To find the dimension for $\mathrm{Sq}(g ; 0, p ; 0, q ; 0)$ 's we want the fundamental group, $F$, for the punctured Riemann sphere to have the maximum number of punctures. Note that Riemann spheres occur at the two middle levels of the square. Let $G$, as in the examples in Section 6 , be $S_{p} \times S_{q}$, and let the square arise from

$$
W_{(p-1)(q-1)}\left(a_{1}, a_{2}, \ldots, a_{2 p-2} ; b_{1}, b_{2}, \ldots, b_{2 q-2}\right)
$$

where the $a$ 's are transpositions generating $S_{p}$, and the $b$ 's are transpositions generating $S_{q}$. So the dimension in moduli space for genus $(p-1)(q-1)$ for such squares is $(2 p-2)+(2 q-2)-3=2 p+2 q-7$. It's codimension in the space of the above Iv's is $(p-1)(q-1)$.

We now give a geometric interpretation for a $\operatorname{Sq}((p-1)(q-1) ; 0, p ; 0, q ; 0)$.

Let $C_{p+q}$ be a plane curve of degree $p+q$ with two ordinary singularities $R_{p}, R_{q}$ of multiplicities $p$ and $q$ (genus $=(p-1)(q-1)$ ). Let $x$ be a point on the curve. A line through $x$ and $R_{p}$ cuts the curve in $q$ points. $q$ lines connect these $q$ points to $R_{q}$. A line through $x$ and $R_{q}$ cuts the curve in $p$ points. $p$ lines connect these $p$ points to $R_{p}$. This set of $p+q$ lines intersect (in general) in $p q$ points. At least $p+q-1$ of these points lie on the curve. 
Definition. $x$ will be called total of all $p q$ points lie on the curve.

THEOREM 8.1. If one point of $C_{p+q}$ is total then all points of $C_{p+q}$ are total.

We will prove this theorem in several steps.

Lemma 8.2. Let $g_{p}^{1}$ be the fibers of the p-sheeted covering in the Iv. Let $g_{q}^{1}$ be the fibers in the $q$-sheeted covering in the Iv. Then $(q-1) g_{p}^{1}$ (and also $\left.(p-1) g_{q}^{1}\right)$ is not special.

Proof. Suppose $(q-1) g_{p}^{1}$ is special. Fix a divisor, $E$, in $K_{g}-(q-1) g_{p}^{1}$. Let $y_{1}$ be a general point in $W_{g}$. Let $y_{1}+y_{2}+\cdots+y_{q}$ be the divisor in $g_{q}^{1}$ containing $y_{1}$. Let $D_{i}, i=2,3, \ldots, q$, be the divisors in $g_{p}^{1}$ containing $y_{i}$. Then $\Sigma D_{i}$ is a divisor in $(q-1) g_{p}^{1}$ not containing $y_{1}$. By Riemann-Roch $y_{1}$ is in $E$ since $g_{q}^{1}$ is always special. This contradiction proves the lemma.

Lemma 8.3. The $\operatorname{CSIv}(g ; 0, p ; 0, q)$ admits a completion if and only if $p g_{q}^{1}$ is equivalent to $q g_{p}^{1}$.

Proof. For notational convenience denote the above $\operatorname{CSIv}$ by $\operatorname{CSIv}(g ; h, p$; $k, q)$ so that $h=k=0$. If this Iv admits a completion $\mathbf{P}^{1}$, then the $g_{1}^{1}$ on $\mathbf{P}^{1}$ lifts to a $g_{q}^{q}$ on $W_{h}$, which lifts in turn to $q g_{p}^{1}$ on $W_{g}$. Lifting $g_{1}^{1}$ thru $W_{k}$ gives a $p g_{q}^{1}$ on $W_{g}$, and so $p g_{q}^{1}$ is equivalent to $q g_{p}^{1}$. For the converse note that $\left|p g_{q}^{1}\right|=$ $g_{p q}^{p+q-1}$ since $p g_{q}^{1}$ is not special. The incomplete $q g_{p}^{1}$ has dimension $q$ and the incomplete $p g_{q}^{1}$ has dimension $p$. Thus there is a $g_{p q}^{1}$ in $g_{p q}^{p+q-1}$ common to both of these incomplete linear series. This $g_{p q}^{1}$ completes the Iv.

Note that this shows that completing the CSIv is equivalent to all points on $C_{p+q}$ being total.

Proof of Theorem 8.1. If one point is total then $p g_{q}^{1}$ is equivalent to $q g_{p}^{1}$. The result follows from the above lemmas.

For $g=4$, the completion of the Iv is equivalent to $3 g_{3}^{1} \equiv 3 h_{3}^{1}$. Since $g_{3}^{1}+h_{3}^{1}$ is canonical this is equivalent to $6 g_{3}^{1}$ being tricanonical. By Riemann's theorem this in turn is equivalent to the theta function vanishing to order 2 at a $1 / 6^{\text {th }}$-period (which is not a half-period).

Alan Landman has shown that Theorem 8.1 is a special case of a theorem where the hypotheses are quite a bit more general and the conclusion is the same.

\section{REFERENCES}

[1] R. D. M. Accola, Plane models for Riemann surfaces admitting certain half-canonical linear series, II, Trans of the AMS 263 (1981), 243-259. 
[2] R. D. M. Accola, Topics in the theory of Riemann surfaces, Lecture notes in mathematics 1595, Springer, 1994.

[ 3 ] R. D. M. Accola, Vanishing thetanulls for some dihedral and cyclic coverings of Riemann surfaces, Kodai Math. J. 28 (2005), 73-91.

[4] Ernst Kani, On Castelnuovo's equivalence defect, Journal fur die Reine und Angewandte Mathematik 352 (1984), 24-69.

[ 5] J. Lewittes, Riemann surfaces and the theta function, Acta Mathematica 111 (1964), 37-61.

DePARTMENT OF MATHEMATiCS

BROWN UNIVERSITY

PROVIDENCE RI, 02912

E-mail: raccola@math.brown.edu 